Artigo Original

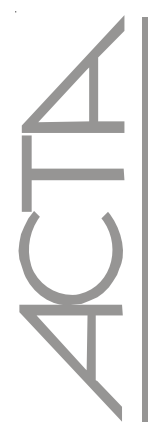

\title{
Diagnósticos de enfermagem de pacientes internadas em unidade médico-cirúrgica*
}

\author{
Nursing Diagnoses in Patients Admitted to a Medical-Surgical Unit \\ Diagnósticos de enfermería de pacientes internadas en una unidad médico quirúrgica

\begin{abstract}
Marcia Paschoalina Volpatoํ, Diná de Almeida Lopes Monteiro da Cruz ${ }^{2}$
\end{abstract}

\begin{abstract}
RESUMO
Objetivo: Identificar os diagnósticos de enfermagem e os Padrões Funcionais de Saúde (PFS) alterados em pacientes hospitalizadas. Método: Roteiro segundo os PFS foi usado para realizar entrevistas, exames físicos e consultas aos prontuários de 60 pacientes (mulheres=100\%; idade média $=49,7 \pm 19,0$ anos) de unidade de internação. Os diagnósticos foram estabelecidos por consenso entre quatro pesquisadores sobre diagnósticos de enfermagem e com experiência em enfermagem médico-cirúrgica. Resultados: Identificaram-se 337 diagnósticos em 48 categorias diferentes. Os diagnósticos mais freqüentes foram: risco de infecção $(58,3 \%)$, dor $(50,0 \%)$, constipação $(41,6 \%)$ e intolerância à atividade $(35,0 \%)$. Os PFS mais representados foram: atividade-exercício (19,9\%); nutricional-metabólico (19,6\%) e percepção e controle da saúde $(18,7 \%)$. Conclusão: O cuidado do paciente internado em clínica médico-cirúrgica exige ampla gama de conhecimentos e habilidades da enfermeira.
\end{abstract}

Descritores: Diagnóstico de Enfermagem; Saúde do adulto; Pacientes internados; Prevalência; Avaliação

\begin{abstract}
Objective: To identify the nursing diagnoses and the altered Functional Health Patterns (FHP) in patients admitted to a medical-surgical unit. Method: A FHP guideline was used to interview, perform physical examinations, and to conduct chart reviews of 60 inpatients (female $=100 \%$; mean age $49.7 \pm 19.0$ year). Nursing diagnoses were declared by consensus among four researchers on nursing diagnoses with expertise on medical-surgical nursing. A total of 337 nursing diagnoses in 48 categories were identified. The most frequent nursing diagnoses were risk for infection $(58.3 \%)$, pain $(50.0 \%)$, constipation $(41.6 \%)$, and activity intolerance $(35.0 \%)$. The most frequent FHP were activity-exercise $(19.9 \%)$, nutritional-metabolic (19.6\%), and health perception-health management (18.7\%). Conclusion: Providing care for the medical-surgical patient requires a wide range of nursing knowledge and skills.
\end{abstract}

Keywords: Nursing diagnosis; Adult health; Inpatients; Prevalence; Evaluation

\section{RESUMEN}

Objetivo: Identificar los diagnósticos de enfermería y los Patrones Funcionales de Salud (PFS) alterados en pacientes hospitalizadas. Método: Para realizar las entrevistas fue utilizado un esquema según los PFS, exámenes físicos y consultas a las historias clínicas de 60 pacientes (mujeres $=100 \%$; edad promedio $=49,7 \pm 19,0$ años) de unidad de internamiento. Los diagnósticos fueron establecidos por consenso entre cuatro investigadores sobre diagnósticos de enfermería y con experiencia en enfermería médico-quirúrgica. Resultados: Se identificaron 337 diagnósticos en 48 categorías diferentes. Los diagnósticos más frecuentes fueron: riesgo de infección (58,3\%), dolor $(50,0 \%)$, constipación $(41,6 \%)$ e intolerancia a la actividad (35,0\%). Los PFS más representados fueron: actividad-ejercicio (19,9\%); nutricional-metabólico $(19,6 \%)$ y percepción y control de la salud (18,7\%). Conclusión: El cuidado del paciente internado en clínica médico-quirúrgica exige una amplia gama de conocimientos y habilidades de la enfermera.

Descriptores: Diagnóstico de enfermería; Salud del adulto; Pacientes internados; Prevalencia; Evaluación

\footnotetext{
* Dissertação de Mestrado apresentado ao Programa de Mestrado Interinstitucional USP/UEL/UEM da Escola de Enfermagem da Universidade de São Paulo - USP - São Paulo (SP), Brasil.

${ }^{1}$ Mestre em Enfermagem; Docente da Universidade Estadual de Londrina. - UEL - Londrina (PR), Brasil.

${ }^{2}$ Professora Associada da Escola de Enfermagem da Universidade de São Paulo - USP - São Paulo (SP), Brasil.
} 


\section{INTRODUÇÃO}

Estudos epidemiológicos que informam sobre a freqüência dos diagnósticos de enfermagem em uma população contribuem para organizar a base de conhecimento de enfermagem, prever os cuidados de enfermagem necessários a uma população específica e orientar a seleção de elementos no serviço e nos programas de educação ${ }^{(1)}$. Dados sobre a prevalência de diagnósticos em populações específicas permitem estimar a probabilidade de as pessoas dessas populações apresentarem determinados diagnósticos, favorecendo a previsão das necessidades de enfermagem, da carga de trabalho do enfermeiro e estimativas dos resultados que podem ser obtidos com os cuidados de enfermagem ${ }^{(2)}$.

No Brasil já se encontram publicações de pesquisas de identificação de diagnósticos em diversas áreas da enfermagem $^{(3-18)}$,e a continuidade de estudos desse tipo permitirá acumular resultados que, integrados, poderão apoiar decisões sobre os focos clínicos nas diferentes áreas clínicas de enfermagem.

Este estudo teve como objetivos descrever os diagnósticos de enfermagem e identificar os Padrões Funcionais de Saúde (PFS) alterados em pacientes hospitalizadas em clínica médico-cirúrgica.

Os PFS formam um conjunto de onze categorias nominais que especificam áreas de saúde pertinentes à enfermagem $^{(19)}$. A descrição e a avaliação dos PFS permitem que as enfermeiras identifiquem os padrões de saúde que são funcionais (forças do cliente) e os padrões de saúde que são disfuncionais (diagnósticos de enfermagem). Além disso, os PFS podem ser usados como forma organizada de avaliação dos dados; como uma estrutura para o agrupamento dos diagnósticos de enfermagem; como um sistema para a organização do conhecimento clínico; como um sistema para a organização da literatura clínica e, por fim, como tópicos para pesquisas clínicas sobre padrões de saúde ${ }^{(1)}$.

\section{MÉTODOS}

O estudo, descritivo e exploratório, foi realizado em um hospital-escola de Londrina, Paraná, em unidade médico-cirúrgica feminina com atendimento a várias especialidades clínicas e cirúrgicas. A unidade dispunha de 46 leitos para a internação de mulheres com idade superior a 12 anos, e a média de internação era de 6 a 8 pacientes por dia.

O estudo obteve parecer favorável do Comitê de Ética da Instituição e todas as participantes assinaram Termo de Consentimento Livre e Esclarecido.

A amostra foi composta por 60 mulheres internadas no período de fevereiro a março de 2000. Para a coleta dos dados foram selecionadas quatro pacientes a cada dia de coleta, por meio de amostragem aleatória simples entre as pacientes com tempo de internação entre $24 \mathrm{e}$ 48 horas. Todas as pacientes do estudo foram avaliadas por entrevista, exame físico e consulta ao prontuário. A coleta dos dados foi orientada por um formulário criado especificamente para esse fim, com base nos PFS propostos por Gordon ${ }^{(1)}$ e realizada entre 24 e 48 horas após a internação da paciente.

Diariamente, com base nos dados coletados das pacientes avaliadas, os diagnósticos de enfermagem foram formulados. Cada doente tinha uma ficha onde eram registradas as informações sobre cada diagnóstico formulado segundo a publicação brasileira de $2000 \mathrm{da}$ classificação da $\mathrm{NANDA}^{(20)}$, incluindo título, características definidoras e fatores relacionados.

Os dados obtidos no instrumento de coleta foram a base para formular os diagnósticos de enfermagem. Dentro de cada PFS, foram relacionados os dados relevantes da paciente. Dado relevante era toda informação que pudesse se constituir em característica definidora ou em fator relacionado de algum diagnóstico. Os dados assim identificados foram agrupados conforme a pesquisadora julgasse haver relação entre eles na composição das características definidoras de possíveis diagnósticos, conduzindo a formulação de hipóteses diagnósticas.

As hipóteses diagnósticas formuladas em cada PFS foram comparadas aos conteúdos da classificação da NANDA $^{(20)}$ para confirmar ou rever os julgamentos iniciais. Cada afirmação diagnóstica constou do título dos diagnósticos e do conjunto das características definidoras. Os fatores relacionados, embora tenham sido sugeridos para vários diagnósticos formulados, não constaram do estudo, porque houve dificuldades, em alguns casos, para identificá-los e, em outros, para confirmar sua pertinência aos diagnósticos.

Com a finalidade de limitar potenciais vieses oriundos de apenas uma interpretação e aumentar a confiabilidade dos diagnósticos formulados, os instrumentos com os dados coletados de cada doente foram submetidos à apreciação de três enfermeiras que possuíam, no mínimo, cinco anos de experiência clínica em enfermagem médicocirúrgica, e com experiência prévia ou atual em pesquisas de identificação de diagnósticos de enfermagem. Essas três colaboradoras, de posse dos registros das coletas dos dados, indicaram, separadamente, os diagnósticos com as respectivas características definidoras que julgaram pertinentes a cada paciente. Com isso, obtiveram-se, para cada paciente, 4 relações de diagnósticos (uma da pesquisadora principal e três das diagnosticadoras). As quatro listas de cada paciente foram analisadas pelas duas autoras deste estudo. Os diagnósticos formulados por pelo menos três das quatro diagnosticadoras foram aceitos e incluídos nos resultados. Os que foram 
formulados por menos que três diagnosticadoras foram revistos pelas autoras, retornando-se ao conjunto dos dados de entrevista e exame físico de cada paciente. Os diagnósticos que não haviam atingido concordância de pelo menos três diagnosticadoras foram incluídos quando as autoras, por consenso, os consideraram suficientemente fundamentados nos dados coletados com base nessa última análise. Destaca-se que o investigador principal foi quem interagiu com as pacientes na coleta dos dados.

\section{RESULTADOS}

As características das 60 pacientes do estudo estão apresentadas na Tabela 1.

Tabela 1 - Características da amostra. Londrina, 2000.

\begin{tabular}{|c|c|c|c|c|c|}
\hline \multirow{2}{*}{ Variáveis } & \multicolumn{2}{|c|}{ Variação } & \multirow{2}{*}{ Méd. } & \multirow{2}{*}{ (DP) } & \multirow{2}{*}{ Mediana } \\
\hline & Máx. & Mín. & & & \\
\hline Idade & 82 & 14 & 49,7 & $(19,0)$ & 49,0 \\
\hline Escolaridade & 14 & - & 3,4 & $(3,6)$ & 2,5 \\
\hline $\begin{array}{l}\text { Renda mensal } \\
\text { (salários mínimos) }\end{array}$ & 8 & - & 2,5 & $(2,0)$ & 2,0 \\
\hline
\end{tabular}

As análises e sínteses das entrevistas e exames físicos das 60 doentes estudadas permitiram estabelecer 337 diagnósticos (48 categorias), com média de 5,6 ( $\pm 2,6)$ diagnósticos por paciente.

A Tabela 2 mostra as 48 categorias de diagnósticos formulados segundo os PFS. Os títulos dos diagnósticos foram lançados conforme a publicação brasileira de 2006 da classificação da NANDA $^{(21)}$.

\section{DISCUSSÃO}

$\mathrm{Na}$ época em que os dados deste estudo foram analisados, a classificação da NANDA $^{(20)}$ incluía 150 diagnósticos de enfermagem e 48 foram identificados na amostra estudada. Esse total correspondia a 32\% das categorias diagnósticas, indicando que as necessidades de cuidados das pacientes da amostra foram bastante variadas.

A natureza dos diagnósticos identificados permite inferir, com base em dados empíricos, qual o domínio de conhecimento necessário ao enfermeiro para organizar o cuidado a essas pacientes.

Os diagnósticos que ocorreram com maior freqüência foram os risco de infecção (58,3\%), dor (50,0\%), constipação $(41,6 \%)$ e intolerância à atividade $(35,0 \%)$, distúrbio no padrão do sono (28,3\%), mobilidade física prejudicada $(26,6 \%)$ e integridade da pele alterada $(26,6 \%)$ (Tabela 2). Esses diagnósticos estão também entre os mais freqüentes em outros estudos com diferentes amostras de pacientes de clínicas médico-cirúrgicas ${ }^{(10,11,22)}$. São identificados, geralmente com freqüências superiores a $50 \%$ das amostras, embora variem sua posição acima dessa freqüência.

A média de diagnósticos por paciente foi de 5,6 e 32 das 48 categorias foram identificadas em no máximo 6 $(10 \%)$ das pacientes (Tabela 2). Esse resultado mostra que a diversidade de necessidades nessa amostra foi intensa. Em estudo junto a pacientes hospitalizados por AIDS em Los Angeles, as pesquisadoras identificaram 45

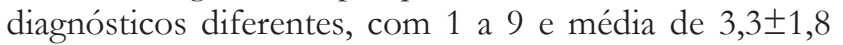
diagnósticos por paciente ${ }^{(23)}$. Em estudo na Islândia em que se analisaram 1217 prontuários, a média de diagnósticos por paciente foi de 3,28, variando 1 a $10^{(24)}$.

A média de diagnósticos de 5,6, observada neste estudo é superior ao observado no estudo da Islândia, pode ser explicada pelo fato de a identificação dos diagnósticos ter sido realizada para fim de pesquisa e não como resultado da prática clínica diária. As pacientes desta pesquisa tinham poucas características em comum, pois estavam internadas por motivos diferentes, o que contribui para explicar a diversidade de diagnósticos com baixa freqüência. Essa diversidade mostra que se requer um conhecimento amplo do profissional que atua em unidades similares a deste estudo desde a fase de identificação dos diagnósticos.

A enfermeira clínica em unidades de internação com clientela semelhante a deste estudo necessita de um conhecimento amplo que a torne capaz de realizar um julgamento clínico eficiente não só na formulação de diversos diagnósticos de enfermagem, mas também na escolha de intervenções adequadas. A escolha adequada de intervenções inclui também a priorização criteriosa dos diagnósticos, o que é essencial especialmente nos casos dos pacientes com grande número de diagnósticos.

Foi grande a variedade de diagnósticos de enfermagem. No entanto, outros mais poderiam ser formulados, se o estudo considerasse todo o processo de internação da paciente. O fortalecimento da interação enfermeiro-paciente após o primeiro contato, permite a observação de outras pistas diagnósticas e, além disso, as próprias intervenções terapêuticas vão gerando novas necessidades de cuidados no decorrer do período de internação.

O Padrão de Atividade-Exercício foi o mais freqüente com 19,9\% dos 337 diagnósticos identificados (Tabela 2). Esse padrão refere-se a exercícios, atividade, lazer, recreação e capacidade de realizar as atividades da vida diária ${ }^{(1)}$. As freqüências dos diagnósticos de intolerância a atividade $(35,0 \%)$ e mobilidade física prejudicada $(26,6 \%)$ foram os que mais contribuíram para a predominância desse padrão (Tabela 2).

O Padrão Nutricional-Metabólico, o segundo mais representado, com 19,6\% do total dos diagnósticos formulados, é definido como o padrão que descreve o consumo de alimentos e líquidos de acordo com as necessidades metabólicas. Inclui os padrões alimentares 
Tabela 2 - Freqüência dos diagnósticos de enfermagem identificados segundo padrões funcionais de saúde em mulheres internadas em unidade médico-cirurgica. Londrina, 2000.

\begin{tabular}{|c|c|c|c|c|}
\hline Padrões & $\% *$ & Diagnósticos ${ }^{* *}$ & n & $\%$ \%** \\
\hline \multirow{10}{*}{ Atividade-exercício } & \multirow{10}{*}{19,9} & Intolerância à atividade & 21 & 35,0 \\
\hline & & Mobilidade física prejudicada & 16 & 26,6 \\
\hline & & Fadiga & 15 & 25,5 \\
\hline & & Déficit no autocuidado para banho/vestir-se & 4 & 6,6 \\
\hline & & Desobstrução ineficaz de vias aéreas & 3 & 5,0 \\
\hline & & Déficit no autocuidado para todas as atividades & 3 & 5,0 \\
\hline & & Padrão respiratório ineficaz & 2 & 3,3 \\
\hline & & Troca de gases prejudicada & 1 & 1,6 \\
\hline & & Risco de troca de gases prejudicada & 1 & 1,6 \\
\hline & & Perfusão tissular ineficaz & 1 & 1,6 \\
\hline \multirow{13}{*}{ Nutricional-metabólico } & \multirow{13}{*}{19,6} & Integridade da pele prejudicada & 16 & 26,6 \\
\hline & & Risco de integridade da pele prejudicada & 11 & 18,3 \\
\hline & & Nutrição desequilibrada: mais do que... & 7 & 11,6 \\
\hline & & Volume de líquidos excessivo & 6 & 10,0 \\
\hline & & Nutrição desequilibrada: menos... & 6 & 10,0 \\
\hline & & Dentição prejudicada & 5 & 8,3 \\
\hline & & Risco de nutrição desequilibrada: menos... & 4 & 6,6 \\
\hline & & Risco de aspiração & 4 & 6,6 \\
\hline & & Mucosa oral prejudicada & 3 & 5,0 \\
\hline & & Risco de amamentação ineficaz & 1 & 1,6 \\
\hline & & Hipertermia & 1 & 1,6 \\
\hline & & Deglutição prejudicada & 1 & 1,6 \\
\hline & & Volume de líquidos deficiente & 1 & 1,6 \\
\hline \multirow{6}{*}{ Percepção e controle da saúde } & \multirow{6}{*}{18,7} & Risco de infecção & 35 & 58,3 \\
\hline & & Risco de trauma & 12 & 20,0 \\
\hline & & Risco de controle ineficaz do tratamento & 12 & 20,0 \\
\hline & & Proteção ineficaz & 2 & 3,3 \\
\hline & & Manutenção ineficaz da saúde & 1 & 1,6 \\
\hline & & Controle ineficaz do regime terapêutico & 1 & 1,6 \\
\hline \multirow{5}{*}{ Eliminação } & \multirow{5}{*}{10,7} & Constipação & 25 & 41,6 \\
\hline & & Incontinência urinária & 6 & 10,0 \\
\hline & & Eliminação urinária prejudicada & 3 & 5,0 \\
\hline & & Risco de constipação & 1 & 1,6 \\
\hline & & Incontinência intestinal & 1 & 1,6 \\
\hline \multirow{5}{*}{ Padrão cognitivo-perceptivo } & \multirow{5}{*}{10,7} & Dor & 30 & 50,0 \\
\hline & & Conhecimento deficiente & 3 & 5,0 \\
\hline & & Percepção sensorial perturbada: tátil & 1 & 1,6 \\
\hline & & Percepção sensorial perturbada: auditiva & 1 & 1,6 \\
\hline & & Confusão aguda & 1 & 1,6 \\
\hline \multirow{5}{*}{ Autopercepção e autoconceito } & \multirow{5}{*}{5,9} & Ansiedade & 14 & 23,3 \\
\hline & & Medo & 3 & 5,0 \\
\hline & & Sentimento de impotência & 1 & 1,6 \\
\hline & & Desesperança & 1 & 1,6 \\
\hline & & Baixa auto-estima & 1 & 1,6 \\
\hline Sono e repouso & 5,0 & Padrão de sono perturbado & 17 & 28,3 \\
\hline Sexual reprodutivo & 4,2 & Padrões de sexualidade ineficazes & 14 & 23,3 \\
\hline Tolerância e resposta ao estresse & 3,3 & Enfrentamento ineficaz & 11 & 18,3 \\
\hline Desempenho de papel e relacionamento & 2,0 & Comunicação verbal prejudicada & 7 & 11,6 \\
\hline \multirow[t]{2}{*}{ Crença e valor } & - & - & - & - \\
\hline & & Número total de diagnósticos & 337 & \\
\hline
\end{tabular}

* Total de diagnósticos no padrão sobre o total geral de diagnósticos (337)

** Títulos conforme a publicação brasileira de 2006 da classificação da NANDA (21)

*** Proporção sobre o número total de pacientes (60) 
do indivíduo, os tipos e quantidades de alimentos consumidos e suas preferências. Estão incluídos nesse padrão as condições da pele, cabelos, unhas, membranas mucosas e dentes, temperatura, peso e altura corporal ${ }^{(1)}$. Observa-se na Tabela 2 que os diagnósticos referentes à integridade da pele foram os que mais contribuíram para a freqüência desse padrão, seguidos pelos diagnósticos referentes às alterações nutricionais e depois pelos relacionados ao volume de líquidos.

O Padrão de Percepção e Controle da Saúde representou 18,7\% do total dos diagnósticos formulados. Esse padrão refere-se à percepção do paciente quanto a sua saúde em geral e quanto à enfermidade atual. Inclui os conceitos do paciente no que se refere às práticas de manutenção da saúde, bem como comportamentos de seguimento de orientações e recomendações médicas e de enfermagem para o controle da enfermidade atual ${ }^{(1)}$. Contribuíram para a freqüência desse padrão especialmente os diagnósticos de risco de infecção, risco de trauma e de risco para controle ineficaz do tratamento (Tabela 2). Esse resultado indica que, além de intervenções de prevenção de riscos durante a internação, a avaliação e intervenção em aspectos relativos à percepção do cliente sobre sua saúde e sobre as práticas de manutenção e controle da sua saúde são prioritárias na área de enfermagem médico-cirúrgica.

Os padrões autopercepção e autoconceito, sono e repouso, sexual-reprodutivo, tolerância e resposta ao estresse, desempenho de papel e relacionamento, apresentaram freqüência de alterações abaixo de 10,0\% (Tabela 2). A freqüência menor de alterações nesses padrões não significa que eles são menos importantes. Com exceção do padrão de sono e repouso, os outros tratam mais diretamente de respostas psicossociais. Como as pacientes estudadas estavam internadas para tratamento de problemas físicos, era esperado que as respostas psicossociais, em termos quantitativos, não assumissem uma posição predominante.

Não foi apresentado nenhum tipo de alteração no padrão de crença e valor, que é definido como os padrões de valores, as metas/objetivos, as convicções (inclusive espiritual) que um indivíduo possui, os quais orientam as escolhas ou tomada de decisões ${ }^{(1)}$. Esse resultado também foi encontrado em outros estudos ${ }^{(4,5,8,13,15,18)}$. Provavelmente, a identificação de características definidoras desses diagnósticos depende de maior tempo de interação com os pacientes, o que traria oportunidade de aprofundar os vínculos de relacionamento terapêutico, necessários para expressões dessa natureza.

\section{CONCLUSÃO}

A avaliação de 60 pacientes internadas em clínica médico-cirúrgica permitiu identificar 337 diagnósticos (média de 5,6+2,6 por paciente), distribuídos em 49 categorias. Dezesseis diagnósticos foram apresentados por mais de $20 \%$ dos pacientes e o mais freqüente foi o risco para infecção $(58,3 \%)$. Vários diagnósticos foram identificados em poucos pacientes.

Os resultados deste estudo contribuem para o delineamento da relevância dos diferentes focos clínicos pertinentes à enfermagem médico-cirúrgica.

Do ponto de vista da prática clínica, os diagnósticos mais freqüentes indicam as necessidades de cuidados mais prováveis entre pacientes internadas nessa unidade e que, por essa razão, deveriam ser focos prioritários de avaliação, diagnóstico e intervenções. O considerável número de diagnósticos que ocorreram em poucos pacientes confirma que os profissionais da enfermagem de unidades médico-cirúrgicas gerais precisam estar preparadas para uma ampla gama de necessidades de cuidados; precisam ser capazes de identificar condições pouco freqüentes, mas que, se identificadas e tratadas, permitirão a individualização do cuidado.

A realização de estudos de prevalência de diagnósticos com outras amostras pertinentes à enfermagem médicocirúrgica e a agregação de seus resultados permitirão melhor delinear conteúdos essenciais para o ensino de enfermagem médico-cirúrgica.

O processo diagnóstico é um processo em que se lida com a incerteza. O desenvolvimento de estudos para identificação de diagnósticos em populações específicas, cujos resultados sirvam de referência para a organização da assistência de enfermagem a essas populações, está sujeito às incertezas desse processo. À medida que os estudos de validação dos diagnósticos forem sustentando os seus conteúdos, essas incertezas serão amenizadas, ou, pelo menos, mais bem conhecidas, favorecendo a aplicação de resultados de estudos de descrição de diagnósticos em populações específicas.

\section{REFERÊNCIAS}

1. Gordon M. Nursing diagnosis: process and application. 3rd ed. St. Louis: Mosby; c1994.

2. Alexander C. Epidemiologic approaches to validation of nursing diagnoses. In: Monograph of the Invitational Conference on Research Methods for Validating Nursing Diagnoses. California: North American Nursing Diagnosis; 1989. p.121-36.

3. Abrão ACFV, Gutiérrez MGR, Marin HF. Utilização do diagnóstico de enfermagem segundo a classificação da NANDA, para a sistematização da assistência de enfermagem em aleitamento materno. Rev Latinoam Enfermagem. 1997; 5(2):49-59.

4. Barros ALBL, Gomes IM. Perfil dos diagnósticos de enfermagem de pacientes internados com infarto agudo do miocárdio. Acta Paul Enfermagem. 2000; 13( N Esp Pt 2):105-8.

5. Cruz DALM, Arcuri EAM. Diagnóstico de enfermagem de pacientes internados por cardiopatia chagásica crônica. Rev 
Esc Enfermagem USP. 1990; 24(2):265-80.

6. Dalri MCB, Rossi LA, Garcia TR, Carvalho EC. Diagnóstico de enfermagem em uma unidade de queimados: análise estrutural dos enunciados. Rev Bras Enfermagem. 1996; 49(1):7-16.

7. Faria AB, Cruz ICF. Diagnósticos de enfermagem em cliente com TCE e em seu familiar e/ou pessoa significativa. Rev Bras Enfermagem. 1996; 49(4): 549-68.

8. Guerriero ALS, Guimarães HCQCP, Maria VLR. Diagnóstico de enfermagem do paciente adulto no primeiro pósoperatório de cirurgia cardíaca. Acta Paul Enfermagem. 2000; 13(2):59-67.

9. Guerriero ALS, Almeida FA, Guimarães HCQCP. Diagnósticos de enfermagem infantil no primeiro pósoperatório de cirurgia cardíaca. Acta Paul Enfermagem. 2003; 16(1):14-21.

10. Jesus CAC, Carvalho EC. Diagnósticos de enfermagem em clientes com alterações hematológicas: uso da Taxonomia I da NANDA. Rev Latinoam Enfermagem. 1997; 5(4):91-9.

11. Lopes RAM, Macedo DD, Lopes MHBM. Diagnóstico de enfermagem mais freqüente em uma unidade de internação de oncologia. Rev Latinoam Enfermagem. 1997; 5(4):3541.

12. Narchi NZ, Gutiérrez MGR. Diagnósticos de enfermagem em mulheres com câncer de mama submetidas à quimioterapia antineoplásica. Acta Paul Enfermagem. 1997; 10(1):33-42.

13. Pasini D, Alvim I, Kanda L, Mendes RSP, Cruz DALM. Diagnósticos de enfermagem de pacientes em unidades de terapia intensiva. Rev Esc Enfermagem USP. 1996; 30(3):50118.

14. Pimenta CAM, Cruz DALM. Câncer e dor: alterações nos padrões de resposta humana. Acta Paul Enfermagem. 1994;
$7(1): 27-34$.

15. Rossi LA, Torrati FG, Carvalho EC, Manfrim A, Silva DF. Diagnóstico de enfermagem do paciente no período pósoperatório imediato. Rev Esc Enfermagem USP. 2000; 34(2):154-64.

16. Silva VM, Araújo TL, Lopes MVO. Evolução dos diagnósticos de enfermagem de crianças com cardiopatias congênitas. Rev Latinoam Enfermagem. 2006; 14(4):5618.

17. Pereira SVM, Bachion MM. Diagnósticos de enfermagem identificados em gestantes durante o pré-natal. Rev Bras Enfermagem. 2005; 58(6):659-64.

18. Suriano MLF, Barros ALBL. Identificação dos diagnósticos de enfermagem mais freqüentes no período perioperatório dos pacientes submetidos a cirurgias cardiovasculares. Acta Paul Enfermagem. 2000; 13(N Esp Pt 2): 98-104.

19. Gordon M. Nursing diagnosis: process and application. 2nd ed. New York: McGraw-Hill; 1987.

20. North American Nursing Diagnosis Association. Diagnósticos de enfermagem da NANDA: definições e classificação 1999-2000. Porto Alegre: Artes Médicas; 2000.

21. North American Nursing Diagnosis Association. Diagnósticos de enfermagem da NANDA: definições e classificação 2005-2006. Porto Alegre: Artmed; 2006.

22. Galdeano LE, Rossi LA, Santos CB, Dantas RAS. Diagnósticos de enfermagem no perioperatório de cirurgia cardíaca. Rev Esc Enfermagem USP. 2006; 40(1):26-33.

23. Smith AR, Chang BL. Nursing diagnoses for hospitalized patients with AIDS. Nurs Diagn. 1996; 7(1):9-18.

24. Thoroddsen A, Thorsteinsson HS. Nursing diagnosis taxonomy across the Atlantic Ocean: congruence between nurses' charting and the NANDA taxonomy. J Adv Nurs. 2002; 37(4):372-81. 